Memory Questionnaire), daily activity, time to next medical intervention, time to next subsequent therapy, safety assessments and OS. At the moment 8 patients are randomized.

Result(s)*

Conclusion*

\section{SCOUT-1: PROSPECTIVE NON-INTERVENTIONAL STUDY IN BRCA/HRD TESTED OVARIAN CANCER PATIENTS ELIGIBLE FOR FIRST-LINE PLATINUM-BASED CHEMOTHERAPY}

${ }^{1}$ El Braicu, ${ }^{2} \mathrm{R}$ Glowik, ${ }^{3} \mathrm{~K}$ Pietzner, ${ }^{4} \mathrm{M}$ Rose, ${ }^{5} \mathrm{P}$ Wimberger, ${ }^{3} \mathrm{~J}$ Sehouli. ${ }^{1}$ North-Eastern German Society of Gynecological Oncology (NOGGO) and Department of Gynecology with Center for Oncological Surgery, Charité-University Medicine of Berlin, Campus Virchow; ${ }^{2}$ AstraZeneca, Wedel, Germany; ${ }^{3}$ North-Eastern German Society of Gynecological Oncology (NOGGO) and Department of Gynecology with Center for Oncological Surgery, CharitéUniversity Medicine of Berlin, Campus Virchow Klinikum, Berlin, Berlin; ${ }^{4}$ Center for Internal Medicine and Dermatology, Department of Psychosomatic Medicine, CharitéUniversitätsmedizin Berlin, 12200 Berlin, Germany., Berlin; ${ }^{5}$ North-Eastern German Society of Gynecological Oncology (NOGGO) and Department of Gynecology and Obstetrics, University Hospital Carl Gustav Carus, TU Dresden, Dresden, Germany

\subsection{6/ijgc-2021-ESG0.442}

Introduction/Background* At initial diagnosis of ovarian cancer (OC), two third of patients have an advanced stage, accompanied by a poor prognosis. Results from landmark trials of maintenance therapy (MTX) with poly ADP ribose polymerase inhibitors (PARPi), especially in tumors associated with homologous recombination deficiency (HRD), like BRCA-mutated tumors, led to the strong recommendation on testing and treatment procedures in the clinical routine in patients with advanced high-grade epithelial OC. The translation of these guidelines into the clinical routine affects drug as well as care management and therefore needs to be evaluated. This study aims to gain new insights into real-world biomarker testing, $1 \mathrm{~L}$ treatment patterns together with outcomes of patients with newly diagnosed advanced OC in Germany and to capture the influence of 1L PARPi MTX on medical routine.

Methodology SCOUT-1 is a German prospective, non-interventional study (NCT04830709; NOGGO ov54) to collect clinical real-world and patient-reported outcome (PRO) data in patients newly diagnosed (first-line) with histologically confirmed advanced (FIGO stage III or IV) high-grade epithelial ovarian, fallopian-tube, or primary peritoneal cancer. Further eligibility criteria include written informed consent, completed surgery (if applicable), eligible for platinum-based chemotherapy, BRCA1/2 mutation tested and willingness/ability to report PROs electronically. Approximately 750 adult eligible patients are planned to be included in up to 80 hospitals or officebased sites. The primary objective is to determine the effectiveness of standard treatment sequences by assessing progression-free survival according to investigator's assessment and evaluation criteria used in routine clinical practice. Secondary goals are shown in figure 1. Further focus is to describe biomarker-testing algorithm, patient selection and to assess patients' QoL, symptoms, needs as well as patients' expectations. Importantly, the SCOUT-1 will help to collect long-term data as patients will be followed for up to 7 years. The study has been approved by ethics committees and recruitment is planned to start in Q2 2021. The estimated primary completion date is Q2 2032.

Sponsor AstraZeneca, in cooperation with North-Eastern German Society of Gynecological Oncology (NOGGO e.V.)

\section{AUDIT OF MANAGEMENT OF ADVANCED OVARIAN CANCERS AT A GYNAECOLOGIC ONCOLOGY DEPARTMENT -AS PER ESGO 2020 UPDATED GUIDELINES}

S Punneshetty ${ }^{*}$, V Thomas, A Sebastian, DS Thomas, A Thomas, RG Chandy, A Peedicayil. Christian Medical College, Gynaecology Oncology, Vellore, India

\subsection{6/ijgc-2021-ESG0.443}

Introduction/Background* The aim of this audit is to assess the compliance of the Department of Gynaecological Oncology, in a tertiary care hospital, India in the management of advanced ovarian cancers, as per the updated European Society of Gynaecology Oncology(ESGO 2020) quality indicators for advanced ovarian cancer

Methodology

Design A retrospective audit 3 cycles of CTX

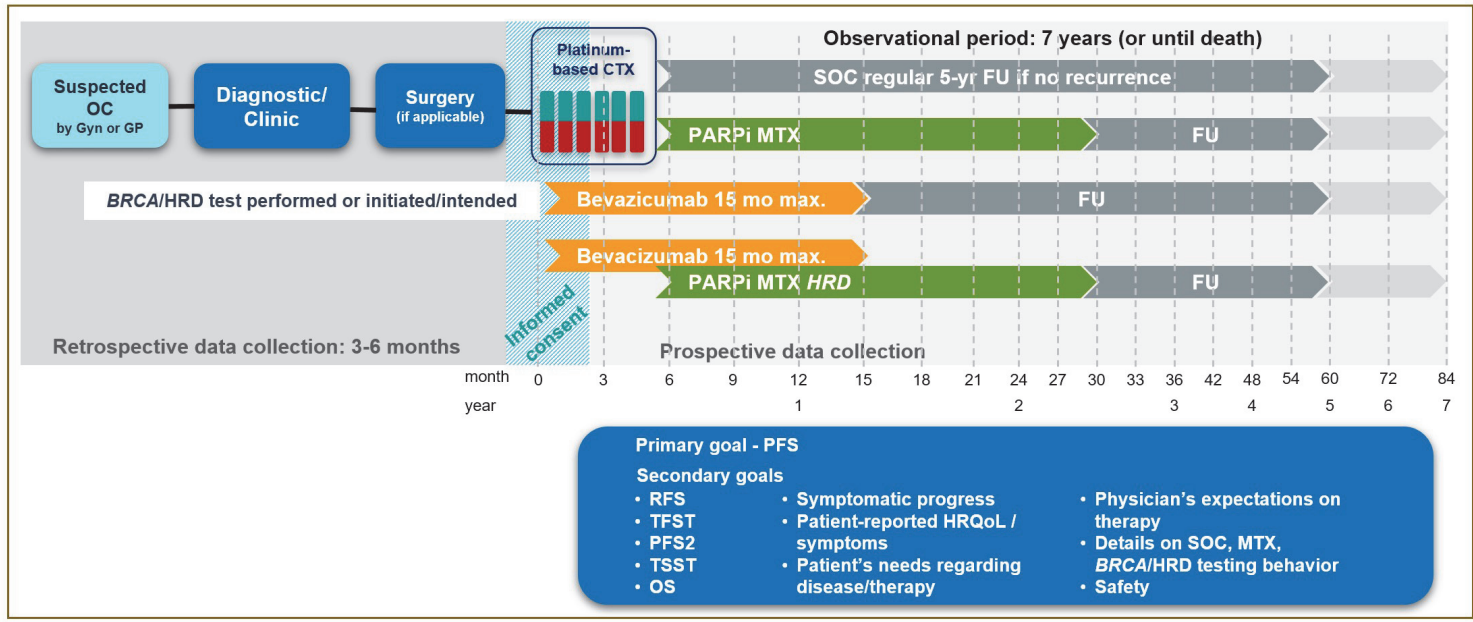


Setting Department of Gynaecologic Oncology, tertiary hospital in India, accredited by ESGO in 2019

Patients Electronic hospital records of 106 patients with advanced ovarian cancer, between Janary 1, 2019 - 31 December 2019 were identified, reviewed and analysed using SPSS Version 21.

Result(s)* Ninety out of 106 patients, underwent cytoreductive surgeries by trained gynaecologic oncologists during this period. The first quality indicator target was not met as the complete resection and primary cytoreductive rates were $49 \%$ and $37 \%$ respectively. Other quality indicators with regard to case load, skills, training and surgeon's experience were fulfilled. Majority (95\%) of the patients were discussed in multi disciplinary board meet. Targets for quality indicators with regard to clinical trial recruitment, preoperative work up and discussion in multidisciplinary board, structured operative report, post-operative complication reporting were achieved. The department has supporting high dependency and intensive care units, but lacks an active perioperative management program. Compliance to pathology reporting was $64 \%$. Overall, a total score of 25 was achieved.

Conclusion* The revised quality indicators laid down by the ESGO helps in introspection and auditing of the department's existing practices for advanced ovarian cancer. The audit revealed the need for judicious selection of patients for primary surgery, improving complete cytoreduction rates and a structured active perioperative management. Synoptic pathologic reporting improves completeness and accuracy.

\section{2 "OVARIAN CANCER IN YOUNG WOMEN": RESULTS IN A COHORT SPANNING 7 YEARS}

LP Castillo Rabazo, B Gil Ibanez*, C Alvarez, G Lopez Gonzalez, MDLR Oliver, L Escudero Villegas, E Felipe Pardo, A Tejerizo. Madrid, Ginecologia y Obstetricia, Hospital 12 de Octubre, Madrid, Spain

\subsection{6/ijgc-2021-ESGO.444}

Introduction/Background* Ovarian cancer is the eighth most frequent cancer worldwide. Although the vast majority of ovarian cancers are diagnosed in postmenopausal women and at advanced stage, a significant subset occurs in younger women. Our objective is to describe the diagnosis and treatment of ovarian cancer in young women in a tertiary Spanish hospital during a 7-year period.

Methodology Retrospective descriptive cohort study of patients aged between 18 and 45 years old diagnosed with ovarian cancer between 01/2012 and 12/2019 at University Hospital 12 de Octubre, Madrid, Spain.

Result(s)* A total of 34 women were included. The mean age at diagnosis was 35.9 years old and the mean body mass index (BMI) was $23.7 \mathrm{~kg} / \mathrm{m}^{2}$. 22/34 (64.7\%) women were nulligesta; 6/34 (17.6\%) had a family history of breast/ovarian cancer; $7 / 34(19.6 \%)$ were smokers and $3 / 34$ (7.8\%) were BRCA mutated.

The mean tumor's size in presurgical imaging workup (ultrasound, CT or MRI scan) was $110.1 \pm 68.5 \mathrm{~mm}$. The mean Ca-125 serum concentration was $128.4 \mathrm{U} / \mathrm{mL}$ in FIGO stages I-II and $393.7 \mathrm{U} / \mathrm{mL}$ in FIGO stages III-IV.

The diagnostic method was adnexectomy in $79.4 \%$ of the patients; 55.9\% performed by laparotomy. 29/34 (85.3\%) patients presented epithelial tumours $(20.6 \%$ low-grade serous, $14.7 \%$ high-grade serous, $17.6 \%$ mucinous, $14.7 \%$ clear cells, $14.7 \%$ endometrioid) and $5 / 34$ non epithelial $(5.9 \%$ dysgerminoma and $11.8 \%$ other non epithelial histology). 23/ $34(67.6 \%)$ patients were diagnosed at FIGO stages I-II.

In patients with FIGO stage III-IV, 4/11 (36.4\%) of patients underwent primary cytoreductive surgery and 6/11 (54.5\%) of patients underwent surgery after neoadjuvant chemotherapy. Complete tumor resection was obtained in $90.9 \%$ of all surgeries. Fertility preservation was possible in $7 / 34$ patients (20.6\%).

Mean follow up time was $48.4 \pm 24.7$ months. Five patients $(14.7 \%)$ relapsed, one patient $(3.4 \%)$ died because of disease and one patient (3.4\%) died because other disease.

At the time of last follow up, 28/34 (82.4\%) women were tumor free, $2 / 34(5.8 \%)$ were alive with disease, $2 / 34(5.8 \%)$ were lost in the follow up.

Conclusion* As reported in literature, women under 45 years old are often diagnosed with early stage ovarian cancer and present optimal survival results with low recurrence rate.

\section{AN INTERNATIONAL SURVEY OF PRACTICE PATTERNS IN OVARIAN CANCER: WHAT WE STAND FOR IN 2021}

${ }^{1,2}{ }^{B}$ Guani*, ${ }^{3}$ L Drouin, ${ }^{4} \mathrm{~T}$ Nikolova, ${ }^{1,2} \mathrm{P}$ Mathevet, ${ }^{5} \mathrm{~F}$ Lécuru, ${ }^{6} \mathrm{H}$ Azais, ${ }^{7} \mathrm{~S}$ Betrian, ${ }^{8} \mathrm{PA}$ Bolze, ${ }^{9} \mathrm{Y}$ Dabi, ${ }^{10} \mathrm{Y}$ Kerbage, ${ }^{11} \mathrm{C}$ Sanson, ${ }^{11} \mathrm{~F}$ Zaccarini, ${ }^{12} \mathrm{~F}$ Guyon, ${ }^{13} \mathrm{C}$ Akladios, ${ }^{14} \mathrm{HC} \mathrm{Hsu},{ }^{9} \mathrm{~S}$ Bendifallah, ${ }^{1} \mathrm{~V}$ Balaya, ${ }^{15} \mathrm{E}$ Deluche. ${ }^{1} \mathrm{CHUV}$, Lausanne, Switzerland; ${ }^{2}$ University of Lausanne, Lausanne, Switzerland; ${ }^{3} \mathrm{CHU}$ de Limoges, Department of Gynecology, Limoges, France; ${ }^{4}$ Academic Teaching Hospital of Heidelberg University, BadenBaden, Germany; ${ }^{5}$ Curie Institut, Breast, gynecology and reconstructive surgery unit, Paris, France; ${ }^{6}$ Georges Pompidou European Hospital, Gynecologic and Breast Oncologic Surgery Department, Paris, France; 7 IUCT Oncopole, Department of medical oncology, Toulouse, France; ${ }^{8}$ Lyon Sud Hospital Center, Pierre-Bénite, France; ${ }^{9}$ Tenon Hospital, Paris, France; ${ }^{10}$ Jeanne de Flandre Hospital, Lille, France; ${ }^{11}$ Gustave Roussy, Villejuif, France; ${ }^{12}$ Institute Bergonié, Bordeaux, France, ${ }^{13}$ Nouvel Hôpital Civil - Hôpitaux Universitaires de Strasbourg, Strasbourg, France; ${ }^{14}$ National Taiwan University Hospital Hsin-Chu Branch, Taiwan; ${ }^{15} \mathrm{CHU}$ de Limoges, Department of medical oncology, Limoges, France

\subsection{6/ijgc-2021-ESG0.445}

Introduction/Background* The aim of this study was to investigate the current surgical and non-surgical therapeutic management of advanced epithelial ovarian cancer (AEOC) cases worldwide, using the data from an internationally launched survey.

Methodology After the validation of a 58-item survey regarding diagnostic and pathological data, as well as surgical and chemotherapeutic strategies of AEOC in France, in the period between April and May 2021, the survey was launched among the members of the following gynecological cancer societies and study groups: IJGC Fellows, SENTICOL 3 study group, ARCAGY-GINECO, AGO, SFOG, SFOG Campus and FRANCOGYN.

Result(s)* A total of 203 physicians completed the survey and majority of them, 171 (84.7\%) were from Europe. Gynecological oncologists represented $49.2 \%$ of the respondents, surgical oncologists $25.1 \%$ and medical oncologists $13.7 \%$. Most of the participants work in centers, managing more than 20 AEOC per year (91.5\%).

According to $66.7 \%$ of respondents, less than $50 \%$ of patients were eligible for primary debulking surgery (PDS). If neoadjuvant chemotherapy (NACT) was used, resectability was assessed after 3 cycles of NACT before interval debulking surgery (IDS) was performed. The most used peritoneal carcinomatosis index was Sugarbaker (69.8\%), whereas Fagotti, modified Fagotti and Makar scores were used in $35.4 \%$, $20.1 \%$ and $4.9 \%$, respectively. 